Objective To describe Olympic-career related significant $(\geq 30$ days duration) injuries.

Design Cross-sectional survey.

Setting The survey was promoted and distributed in eight languages, worldwide via email and social media to Olympians who competed at a Summer and/or Winter Olympic Games and considered themselves retired from Olympic level training and competition.

Patients (or Participants) 3,357 Olympians (44\% female), median age 44.7 yrs (16-97) from 131 countries and 57 Olympic Sports (42 summer, 15 winter), mean 1.6 \pm 0.9 Olympic Games per Olympian.

Interventions (or Assessment of Risk Factors) Olympic-career participation and significant injury history.

Main Outcome Measurements Injury prevalence by sport and anatomical region.

Results There were 3,746 injuries reported in 2,116 Olympians equating to $63.0 \%$ of Olympians (female $68.1 \%$, male $59.2 \%$; Summer 62.0\%, Winter 69.0\%) reporting at least one significant Olympic-career related injury. Overall, 1.1 significant injuries per Olympic-career were reported, with $63.8 \%$ $(n=2389)$ of injuries occurring in training. By sport (Summer and Winter, respectively), injury prevalence was highest in handball (82.2\%), badminton (78.4\%) and judo (77.2\%), and alpine skiing (82.4\%), freestyle skiing (81.6\%), and snowboarding (77.3\%), and lowest for shooting (40.0\%) and swimming (48.5\%), and biathlon (40.0\%) and curling (54.3\%) (sports with $\mathrm{n} \geq 20$ participants). The knee (20.6\%), followed by the lumbar spine (13.1\%), and shoulder (12.9\%) were the most common affected injury locations.

Conclusions Overall, almost two thirds of Olympians reported sustaining at least one significant Olympic-career related injury. Similar to prospective injury studies, injury prevalence varied across sports, with the knee, lumbar spine and shoulder most commonly affected. It is important to understand the nature and causes of injuries during the entire career of an elite athlete, in order to better inform injury prevention and future athlete health initiatives.

\section{THE EPIDEMIOLOGY OF INJURY AND ILLNESS OF ATHLETES AT THE INDIAN OCEAN ISLAND GAMES 2019}

\begin{abstract}
${ }^{1,2}$ Daniel Garnett, ${ }^{3}$ Chandra Adisha Bholah, ${ }^{3}$ Yannick D'Hotman, ${ }^{3}$ Krsna Sunassee, ${ }^{4} J o n$ Patricios, ${ }^{5}$ Benita Olivier, 'Saul Cobbing. 'Physiotherapy Department, College of Health Sciences, University of KwaZulu Natal, Durban, South Africa; ${ }^{2}$ Department of Physiotherapy, Faculty of Health Sciences, University of Pretoria, Pretoria, South Africa; ${ }^{3}$ Comité d'Organisation de la 10ème Edition des Jeux des lles de I'Océan Indien (COJ 2019), Port Louis, Mauritius; ${ }^{4}$ Wits Sport and Health (WiSH), School of Clinical Medicine, Faculty of Health Sciences, University of the Witwatersrand, Johannesburg, South Africa; ${ }^{5}$ Physiotherapy Department, Faculty of Health Sciences, University of the Witswatersrand, Johannesburg, South Africa
\end{abstract}

\subsection{6/bjsports-2021-IOC.57}

Background The Indian Ocean Island Games is a multi-sport event that occurs every four years and includes athletes from seven islands of the Indian Ocean, namely, Comoros, Reunion, Mayotte, Madagascar, Maldives, Seychelles, and Mauritius.

Objective This study aims to describe the injury and illness epidemiology of the athletes participating during the 2019 Indian Ocean Islands Games.

Material and Methods This prospective cohort study recorded injury and illness cases from athletes who competed in these Games. All medical physicians received detailed instructions and training on data collection using an injury report form. All athletes (minor and adults) who provided consent, or consent given from the minors' guardians, were included in this study. Athletes who did not provide consent for this study were excluded.

Results 1521 athletes (531 women and 990 men) reported 160 injuries (injury incidence rate of $10.5 \%$ ) and 85 illnesses (illness incidence rate of 6\%). The percentage of distribution of injuries were highest in football and basketball. Most injuries occurred during competition compared with training Joint sprains were the most common type of injury (28\%), followed by muscle strains (19\%). Men suffered most injuries (79\% vs. $21 \%)$. Similarly, men sustained more illness than women $(57 \%$ vs. $43 \%)$. Most illnesses affected the respiratory system (67\%), and infection was the most common cause of illness $(84 \%)$ in participating athletes.

Discussion These findings are similar to previous events in other parts of the world. However, unique ailments, not previously reported on, were discovered.

Conclusion Epidemiological data from this study can be inferred to athletes who compete in similar multi-sport events and/or Olympic Games in the Indian Ocean region.

\section{AVERAGE RACE DAY ENVIRONMENTAL DATA UNDERESTIMATES INDIVIDUAL ATHLETE ENVIRONMENTAL EXPOSURE IN A MASS- PARTICIPATION ENDURANCE CYCLING EVENT WITH A STAGGERED START: A SAFER STUDY IN 97946 CYCLISTS}

${ }^{1}$ Sonja Swanevelder, ${ }^{2,3}$ Martin Schwellnus, ${ }^{2,3}$ Nicola Sewry, ${ }^{1,4}$ Esme Jordaan. ${ }^{1}$ Biostatistics Unit, South African Medical Research Council, Cape Town, South Africa; ${ }^{2}$ Sport, Exercise Medicine and Lifestyle Institute (SEMLI), Pretoria, South Africa; ${ }^{3}$ IOC Research Centre, South Africa, Pretoria, South Africa; ${ }^{4}$ Statistics and Population Studies Department, University of the Western Cape, Cape Town, South Africa

\subsection{6/bjsports-2021-IOC.58}

Background Race starting time is often staggered over several hours in large mass-participation endurance sports events. Athlete exposure to environmental conditions changes as wetbulb-globe-temperature (WBGT) changes during race day. Slower participants may be exposed to changing environmental conditions for a longer duration. In most studies environmental conditions are reported using an average WBGT for the race day.

Objective To calculate individual average WBGT (iWBGTavg) for each race participant and compare (iWBGTavg) to the average WBGT on race day (WBGTavg).

Design Retrospective, cross-sectional study.

Setting Cape Town Cycle Tour (109 km), South-Africa, 20122014.

Participants Race starters ( $\mathrm{n}=97946)$.

Assessment of Risk factors WBGTavg for each year was calculated using the data over 11hours (race start at 6am; cut-off at $5 \mathrm{pm}$ ) from the weather station at the geographical midpoint of the race. iWBGTavg for each race starter was calculated using individual start times, finishing times and data from automated weather stations on route. Factors that possibly affect the variation in measurement of WBGT are individual staggered start category (start time) $(<07 \mathrm{~h} 00$, 07h0108h00, 08h01-09h00, >09h00) and total race exposure category (hours) (<3hr45min; $3 \mathrm{hr} 45 \mathrm{~min}-4 \mathrm{hr} 30 \mathrm{~min}$; $4 \mathrm{hr} 31 \mathrm{~min}-$ $5 \mathrm{hr} 30 \mathrm{~min}$; $>5 \mathrm{hr} 30 \mathrm{~min})$. 
Outcome measurements Difference between the two WBGT variables (WBGTdiff=iWBGTavg-WBGTavg) for start time and total race exposure (hours) categories (regression modelling).

Results There was a significant greater WBGTdiff $(\mathrm{p}<0.0001)$ in later start time categories (WBGT diff $>$ in categories $07 \mathrm{~h} 01-08 \mathrm{~h} 00=0.38,08 \mathrm{~h} 01-09 \mathrm{~h} 00=0.86$, and $>09 \mathrm{~h} 00=1.17$ vs. reference $<07 \mathrm{~h} 00)$. Similarly, there was a significant greater WBGTdiff $(\mathrm{p}<0.0001)$ in the longer race exposure categories (WBGT diff $>$ in categories $3 \mathrm{hr} 45 \mathrm{~min}-$ $4 \mathrm{hr} 30 \mathrm{~min}=0.24 ; 4 \mathrm{hr} 31 \mathrm{~min}-5 \mathrm{hr} 30 \mathrm{~min}=0.51 ;>5 \mathrm{hr} 30 \mathrm{~min}=0.56$ vs. reference $<3 \mathrm{hr} 45 \mathrm{~min})$.

Conclusions Average race day WBGT underestimated environmental risk exposure in an endurance sports event characterized by staggered race start times and wide variations in individual total race times. We suggest that individualized environmental risk exposure be used in future studies relating risk of medical encounters to environmental exposure.

\section{YEARS OF TRAINING, OLDER AGE, HISTORY OF CHRONIC DISEASE AND MEDICATION USE ARE RISK FACTORS ASSOCIATED WITH OVERUSE INJURIES IN RECREATIONAL CYCLISTS: A CROSS-SECTIONAL SAFER STUDY IN 21824 CYCLISTS}

${ }^{1}$ Francois du Toit, 2,3 Martin Schwellnus, ${ }^{1}$ Paola Wood, ${ }^{4}$ Sonja Swanevelder, ${ }^{2}$ Jannelene Killops, ${ }^{4,5}$ Esme Jordaan. 'Sport, Exercise Medicine and Lifestyle Institute (SEMLI) and Division of Biokinetics and Sports Science, Department of Physiology, Faculty of Health Sciences, University of Pretoria, South Africa, Pretoria, South Africa; ${ }^{2}$ Sport, Exercise Medicine and Lifestyle Institute (SEMLI), South Africa, Pretoria, South Africa; ${ }^{3}$ IOC Research Centre, South Africa, Pretoria, South Africa; ${ }^{4}$ Biostatistics Unit, South African Medical Research Council, South Africa, Cape Town, South Africa; ${ }^{5}$ Statistics and Population Studies Department, University of the Western Cape, South Africa, Cape Town, South Africa

\subsection{6/bjsports-2021-IOC.59}

Background Risk factors associated with overuse injuries in cyclists (OICs) in recreational cyclists have not been well-studied. Objective To determine risk factors associated with OICs in recreational cyclists participating in a mass community-based cycling event.

Design Cross-sectional study.

Setting Cape Town Cycle Tour (CTCT) 2016.

Patients (or Participants) 21824 consenting cyclists $(60.8 \%$ of 35914). Race entrants $(n=35914)$ completed an online prerace medical screening questionnaire.

Interventions (or Assessment of Risk Factors) The online prerace medical screening questionnaire identified 617 cyclists with a history of OICs. Categories of possible risk factors that were explored included demographics, training/racing history, history of chronic disease, and medication use.

Main Outcome Measurements Prevalence ratio (PR) of OICs were explored using multi-variate analyses.

Results The PR of OICs was similar in male and female cyclists, but was significantly higher in older ( $>50$ years) cyclists (vs. $\leq 30$ yrs $\mathrm{PR}=1.6$; vs. 31 to $\leq 40$ yrs $\mathrm{PR}=1.5$; vs. 41 to $<50$ yrs $\mathrm{PR}=1.4 ; \mathrm{p}<0.0001$ vs. age categories). Independent risk factors associated with OICs (adjusted PR for gender and age) were: 1) training/racing variables [increased weekly training/racing $(P R=1.1, p=0.0003)$, increased years of participation in cycling events of $>2$ hours $(\mathrm{PR}=1.1$, $\mathrm{p}=0.0189)$, higher average racing speed category $(\mathrm{km} / \mathrm{h})$ $(\mathrm{PR}=1.04, \mathrm{p}=0.0368)$ ], 2) chronic disease history [symptoms of cardiovascular disease $(\mathrm{PR}=2.3, \mathrm{p}=0.0026)$, respiratory disease $(P R=1.6, p<0.0001)$, nervous system/psychiatric disease
$(\mathrm{PR}=1.5, \quad \mathrm{p}=0.0082)$, and gastrointestinal tract disease $(\mathrm{PR}=1.4, \quad \mathrm{p}=0.0225)$ ], and 3) medication use [prescribed medication use $(\mathrm{PR}=1.2, \mathrm{p}=0.0226)$, analgesic/anti-inflammatory medication used before or during racing $(\mathrm{PR}=5.1, \mathrm{p}<0.0001)]$. Conclusions Increased training, years of participation in endurance cycling events, older age, chronic disease and medication use are novel independent risk factors associated with OICs. However, the causal relationship, including the direction, between OICs and these factors need to be established, before considering these in the design and implementation of prevention programs.

\section{INDEPENDENT RISK FACTORS ASSOCIATED WITH INJURY-RELATED MEDICAL ENCOUNTERS DURING A 109 KM CYCLING EVENT ARE FEMALE SEX, OLDER AGE, FASTER CYCLING SPEED AND ENVIRONMENTAL CONDITIONS: A SAFER STUDY IN 102251 RACE STARTERS}

1,2 Jannelene Killops, ${ }^{3,4}$ Nicola Sewry, ${ }^{1,4}$ Martin Schwellnus, ${ }^{5}$ Sonja Swanevelder ${ }^{1}$ Christa Janse van Rensburg, ${ }^{5,6}$ Esme Jordaan. ${ }^{1}$ Sport, Exercise Medicine and Lifestyle Institute (SEMLI) and Section Sports Medicine, Pretoria, South Africa; ${ }^{2}$ Mediclinic Southern Africa, Stellenbosch, South Africa; ${ }^{3}$ Sport, Exercise Medicine and Lifestyle Institute (SEMLI), Pretoria, South Africa; ${ }^{4}$ IOC Research Centre, South Africa, Pretoria, South Africa; ${ }^{5}$ Biostatistics Unit, South African Medical Research Council, Cape Town, South Africa; ${ }^{6}$ Statistics and Population Studies Department, University of the Western Cape, Cape Town, South Africa

\subsection{6/bjsports-2021-IOC.60}

Background Injury-related medical encounters (injMEs) are common in mass community-based participation cycling events, but there are limited data on the risk factors associated with injuries in endurance cycling events.

Objective To determine the risk factors associated with injMEs in a mass community-based endurance cycling event.

Design Retrospective, cross-sectional study.

Setting Cape Town Cycle Tour (109 km), South Africa.

Participants 102251 race starters.

Assessment of Risk Factors All injMEs (and a subgroup of serious injMEs) for the 3 years were recorded by race medical doctors and nurses and grouped into main anatomical area of injury. The following possible risk factors associated with injMEs were explored: sex, age, cycling speed and environmental exposure [calculated as average individual Wet-Bulb Globe Temperature (iWBGTavg)].

Main Outcome Measures Independent risk factors associated with injMEs, serious injMEs, and injMEs by main anatomical areas using a Poisson regression model.

Results For all injMEs, the independent risk factors associated during an endurance cycling event were: sex (women vs men $p<0.0001)$, older age $(p=0.0005)$, faster cycling speed $(\mathrm{p}<0.0001)$ and higher average individualised Wind Speed (aiWindSpeed, $\mathrm{p}<0.0001$ ). The only risk factor for serious/life-threatening injuries was women $(p=0.0413)$ For specific main anatomical areas the risk factors were: head/ neck (women), upper limb (women,older age, faster cyclists), trunk (women, higher aiWindSpeed) lower limb (higher aiWindSpeed).

Conclusion Women, older age, faster cycling speed higher aiWindSpeed were all risk factors for acute injuries during an endurance cycling event. These risk factors should help inform race organisers and medical teams on race day to ensure the best medical care is given, and effective acute injury prevention programmes are disseminated. 